\title{
Centrosome Dysfunction and Senescence: Coincidence or Causality?
}

\author{
Delowar Hossain ${ }^{1,2}$ and William Y. Tsang ${ }^{1,2,3 *}$
}

${ }^{1}$ Institut de recherches cliniques de Montréal, Canada

${ }^{2}$ Division of Experimental Medicine, McGill University, Montréal, Canada

${ }^{3}$ Faculté de Médecine, Université de Montréal, Montréal, Canada

\begin{abstract}
Centrosomes are the tiny organelles found in most eukaryotic systems. By virtue of their ability to anchor, organize and nucleate microtubules, they play a crucial role in establishing spindle bipolarity and in ensuring the fidelity of cell division. Defects in centrosome structure and function often result in mitotic catastrophe, cell cycle arrest, cell death, genomic instability and/or aneuploidy, leading to human disorders such as primary microcephaly, cancer and ciliopathies. Interestingly, genomic instability and aneuploidy are also hallmarks of aging and cellular senescence, but our understanding of the connection between centrosome dysfunction and senescence remains rudimentary. In this review, we focus on existing evidence suggesting that these two phenomena are indeed related, along with the emerging view that centrosome aberrations represent a form of cellular stress that is necessary and sufficient to trigger a permanent cell cycle arrest and senescence. The molecular mechanisms underlying cellular senescence as a consequence of centrosome aberrations and the involvement of p53 will be discussed.
\end{abstract}

Keywords: Centrosomes; Centrioles; PCM; Aging; Senescence; Genomic instability; Aneuploidy; Stress; p53; Phosphorylation

Abbreviations: Pericentriolar matrix (PCM), Retinoblastoma (Rb)

\section{Review}

\section{Centrosome structure and function}

Although originally discovered and described by Flemming, Van Beneden and Boveri in the late 1800s as a tiny cellular organelle, the centrosome is a remarkably complex structure with diverse functions [1-4]. It is composed of a pair centrioles surrounded by an amorphous cloud of proteins called the pericentriolar matrix (PCM) (Figure 1). Each centriole is made up of nine triplet of stabilized microtubules arranged in a cylindrical manner. The two centrioles are termed the mother and daughter centrioles, and can be distinguished by the presence of sub-distal and distal appendages at the mother centriole. While sub-distal appendages anchor cytoplasmic microtubules, distal appendages are believed to be important for the formation of cilia, cellular antennae possessing motility and/or sensory function $[5,6]$. Centrioles are responsible for organizing the PCM, the major site of microtubule nucleation from which cytoplasmic microtubules emanate and elongate. In addition, there are centriolar satellites, small and granular structures that cluster around the centrosome and participate in microtubule-dependent protein trafficking towards the organelle $[7,8]$. The centrosome coordinates all microtubulerelated functions, including cell division, cell shape, polarity, motility and adhesion.

The number of centrosomes within a cell is tightly regulated during the cell cycle (Figure 1). A single centrosome duplicates once in the S phase, and the two centrosomes, once separated, migrate to opposite poles of a cell and establish the bipolar spindle in mitosis. A functional bipolar spindle ensures faithful chromosome segregation, wherein each incipient daughter cell receives one centrosome and a diploid set of chromosomes. Perturbations known to disrupt centrosome structure and function often have deleterious consequences. For instance, abnormal cell division in mitosis can result in genomic instability and aneuploidy which are characteristics of many types of cancer. In other cases, abnormal mitosis can trigger programmed cell death and impair spindle alignment of neural progenitor cells, leading to their depletion and limiting the total number of neurons that can be generated. These are believed to be the underlying mechanisms responsible for reduced brain size in patients with primary microcephaly and Seckel syndrome. Furthermore, defects in cilia formation and function can cause in a wide variety of human diseases collectively known as ciliopathies. For a general review of the role of centrosomes and cilia in human disorders, we direct the reader to several excellent review articles [9-11].

\section{Centrosomes and senescence: are they related?}

Senescence or aging is a biological process found in all living organisms and is characterized by changes that disrupt cellular metabolism and function with time, resulting in progressive deterioration, cell cycle arrest and cell death. Although observed at the level of the whole organism (in vivo) $[12,13]$ and individual cells (cellular senescence) [14], the molecular and cellular basis of senescence are not fully understood. At a cellular level, it is well-established that senescence is associated with chromosomal instability and aneuploidy in different cell types from various species [15-19]. Several physiological stresses are thought to contribute to the aging process, including the shortening of telomeres [20-22], oxidative stress [23,24], DNA damage [25,26], over-expression of tumor suppressor genes [27] and strong oncogenic signaling [28-30]. Interestingly, increasing evidence also supports a link between centrosome dysfunction and senescence, suggesting that this organelle could directly or

*Corresponding author: William Y. Tsang, Research Unit Director, Cell Division and Centrosome Biology, Institut de recherches cliniques de Montréal, Québec H2W 1R7, Canada, Tel: (514) 987-5719; Fax: (514) 987-5685; E-mail: william tsang@ircm.qc.ca

Received December 02, 2013; Accepted December 04, 2013; Published December 09, 2013

Citation: Hossain D, Tsang WY (2013) Centrosome Dysfunction and Senescence: Coincidence or Causality? Aging Sci 1: 113. doi: 10.4172/2329-8847.1000113

Copyright: (c) 2013 Hossain D, et al. This is an open-access article distributed under the terms of the Creative Commons Attribution License, which permits unrestricted use, distribution, and reproduction in any medium, provided the original author and source are credited. 


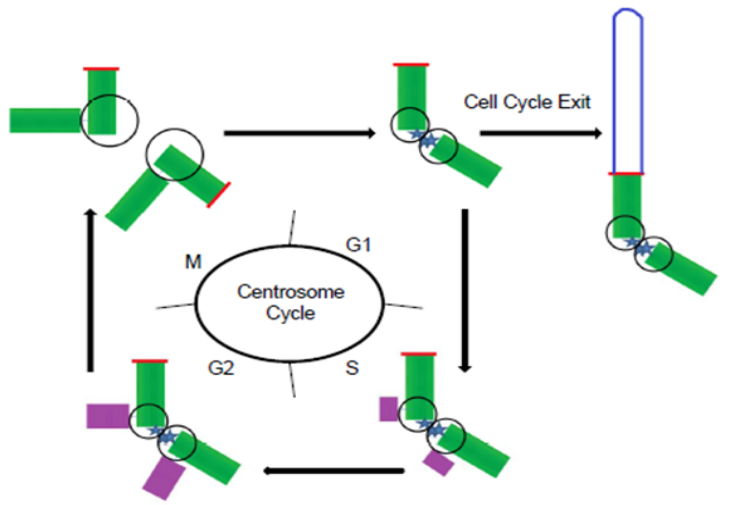

Figure 1: Centrosome structure and biogenesis. A cell in the G1 phase possesses one centrosome consisting of the mother (green rectangle with a red line) and daughter (green rectangle) centrioles surrounded by the PCM (black circle). This single centrosome duplicates in the S phase, leading to the synthesis of two new centrioles, or procentrioles (purple rectangle) adjoining the existing mother and daughter centrioles. Procentrioles elongate and mature into daughter centrioles, while the existing daughter centriole matures into a new mother centriole during $S$ and G2 phases. The PCM becomes enlarged at the onset of mitosis, signifying competence of the two centrosomes in anchoring and organizing microtubules for cell division. After mitosis, each daughter cell can enter the $\mathrm{G} 1$ phase and progress through another round of cell division, or it can exit the cell cycle, in which case a cilium (blue) is formed from the mother centriole.

indirectly play a role in aging. Aged porcine oocytes exhibit a loss of $\gamma$-tubulin and NuMA, critical components of the PCM at the meiotic spindle, giving rise to abnormal and disorganized spindles [31]. Similarly, microtubules are gradually lost from the spindle of aged mouse oocytes, a feature highly suggestive of compromised centrosome structure and function [32,33]. A loss of centrosome and microtubule integrity has also been described in aged human oocyte, both in vitro and in vivo [34], and in aged Drosophila cells [35]. Furthermore, human primary fibroblasts are known to stop dividing permanently after a finite number of cell divisions as a result of telomere shortening and oxidative stress and enter a state of replicative senescence [14]. As these cells age, the frequency of abnormal mitotic figures increase, and this is accompanied by an increase in supernumerary centrosomes [16]. Primary mouse embryonic fibroblasts also undergo replicative senescence with age due to oxidative stress $[23,24]$; however, unlike the situation in human fibroblasts, centrosomes do not increase in number but instead fragment into smaller pieces with increasing passage [36]. Most importantly, the authors showed that disruption of core PCM components in early-passage mouse embryonic fibroblasts can also induce centrosome fragmentation and trigger premature entry to senescence [36], suggesting that centrosome dysfunction alone is sufficient to provoke the induction of a cellular senescence program. Taken together, these studies raise the intriguing possibility that centrosome aberrations, similar to oxidative stress and telomere shortening, is a type of cellular stress that can predispose cells to permanent cell cycle exit, and future studies using high-resolution and electron microscopy will be necessary to define the precise nature of these structural aberrations and the extent to which they contribute to senescence.

\section{Centrosome aberrations, senescence and p53}

Several recent studies have begun to address whether centrosome dysfunction can indeed trigger cellular senescence and whether the underlying molecular pathways overlap with those induced by other physiological stresses. Depletion of a number of centriolar (C-Nap1, $\delta$-tubulin, $\varepsilon$-tubulin), PCM (pericentrin, $\gamma$-tubulin, GCP-2, GCP-3, GCP-5, AKAP450) and centriolar satellite (PCM-1) proteins leads to a loss of centrosome integrity and cell cycle arrest in the G1 phase [37]. The G1 arrest phenotype can be induced in post-mitotic cells, indicating that it is not a consequence of mitotic defects. Prior to the G1 arrest, p38, a protein implicated in cellular stress response and senescence, becomes activated and phosphorylates p53 at Ser33 (and not at Ser15; see below), causing p53 to accumulate at centrosomes before its translocation to the nucleus. Another study also highlighted a role of pericentrin and PCM-1 in cell cycle regulation [38]. Inhibition of pericentrin or PCM-1, which recruits pericentrin to the PCM, induces a permanent cell cycle exit with a concomitant increase in cellular $\beta$-galactosidase expression, a hallmark of cellular senescence. Similar to the previous study, this arrest is also dependent on p38 and p53, and probably occurs as a result of up-regulation of p53 and p 21 protein levels and down-regulation of phosphorylated retinoblastoma ( $\mathrm{Rb})$. Likewise, depletion of other PCM components, including Cep192 (which recruits NEDD1 to the PCM) and NEDD1 (which recruits $\gamma$-tubulin to the PCM), causes centrosome fragmentation and premature entry to senescence [36]. Furthermore, inhibition of Aurora A or its downstream target TACC3, both of which are localized to the PCM during mitosis, leads to premature senescence in p53-proficient tumor cells, characterized by an increase in p53, p21 and hypophosphorylated $\mathrm{Rb}[39,40]$. The elevation in p53 levels could be explained in part by the fact that Aurora A normally phosphorylates p53 at Ser315 to sensitize it for degradation and, in the absence of Aurora A, p53 becomes stabilized [41]. In primary human fibroblasts, cells that have undergone either replicative senescence or premature senescence induced by oxidative stress also accumulate p53 at the centrosome, accompanied by phosphorylation at Ser15 [42]. Ser15 phosphorylation on p53 is essential for its localization to the centrosome, and has been shown to be a default pathway carried out by ataxia telangiectasia mutated, or ATM, at the centrosome in early mitosis to insure correct cell division [43-45]. When the mitotic spindle is correctly in place, Ser15 phosphorylation is rapidly removed and p53 becomes sequestered at the centrosome in an inactive form. On the other hand, when the spindle is impaired, p53 remains phosphorylated at Ser15, and this phosphoprotein is eventually translocated to the nucleus to induce cell cycle arrest and cellular senescence. Therefore, it seems plausible that in response to centrosome damage and possibly other stresses, one key event that takes place early in the senescence process is the phosphorylation and accumulation of p53 at the centrosome. It has been known for a long time that $\mathrm{p} 53$ localizes to centrosomes, but surprisingly little is known about its function at this organelle [42,43,46-48]. It would be interesting in the future to delineate the functional significance of centrosomal p53 and its differential phosphorylation by various kinases, as elucidating these molecular events would undoubtedly provide a better understanding of how p53 integrates signals from different types of stresses, including centrosome dysfunction, to promote cellular senescence.

\section{Conclusions and Perspectives}

The role of centrosomes in aging is an important area of research that has been largely overlooked. Despite little and fragmentary evidence, existing data strongly favor the view that centrosome dysfunction is connected to cellular senescence. We propose that in addition to existing known cellular stresses, 


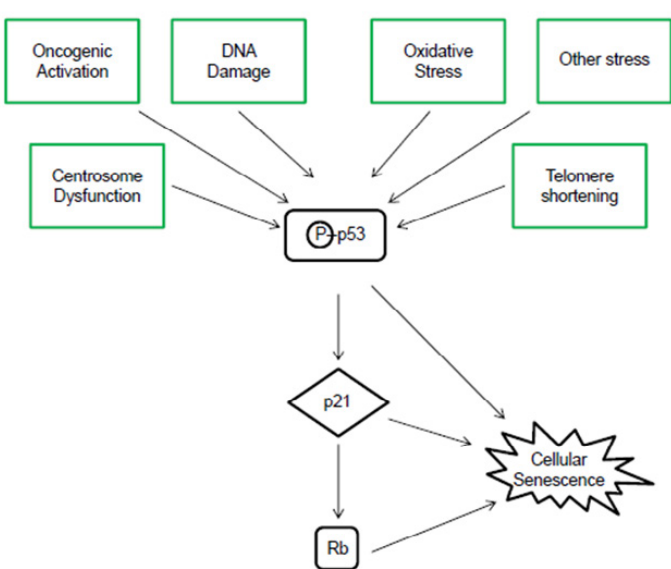

Figure 2: Molecular pathways of cellular senescence. Several types of stresses can predispose cells to undergo senescence. These stresses stimulate cellular signaling pathways resulting in the activation and accumulation of phosphorylated p53 (P-p53) at the centrosome. Phosphorylation of centrosomal p53 subsequently triggers the activation of p21 and Rb (hypo-phosphorylated Rb), and together these three proteins modulate the onset of senescence.

including telomere shortening, oxidative stress, DNA damage, over-expression of tumor suppressors and oncogenic activation, centrosome dysfunction is another form of stress that can predispose cells to cell cycle exit and senescence (Figure 2). In addition, we speculate that these diverse pathways converge on p53. Depending on the source(s) of stress, p53 is promptly accumulated at the centrosome and becomes phosphorylated on different residues by different kinases. Phosphorylation of centrosomal p53 is subsequently needed to fine-tune downstream events, such as the activation of $\mathrm{p} 21$ and $\mathrm{Rb}$, ultimately leading to permanent cell cycle arrest and cellular senescence. While much is known about the role of nuclear p53 as a transcriptional regulator, its biological function at the centrosome warrants further investigation. For instance, what are the molecular mechanisms by which p53 is shuttled into and out of the centrosome, and how does the spatial and temporal regulation of p53 phosphorylation modulate its localization and function? Above all, what is the precise role of centrosomally localized p53 in senescence? We believe that answers to these questions should catalyze an exciting wave of research studies into the interconnections between cellular senescence, cell death, and uncontrolled cell growth, critical biological process that are inextricably linked to proper centrosome function.

\section{Acknowledgement}

We apologize to our colleagues whose findings could not be included due to space limitations. W.Y.T. was a Canadian Institutes of Health Research New Investigator and a Fonds de recherché Santé Junior 1 Research Scholar This work was supported by the Natural Sciences and Engineering Research Council of Canada Discovery Grant to W.Y.T. D.H. was supported by a Graduate Excellence Award from McGill University.

\section{References}

1. Debec A, Sullivan W, Bettencourt-Dias M (2010) Centrioles: active players or passengers during mitosis? Cell Mol Life Sci 67: 2173-2194.

2. Nigg EA, Stearns T (2011) The centrosome cycle: Centriole biogenesis, duplication and inherent asymmetries. Nat Cell Biol 13: 1154-1160.

3. Azimzadeh J, Marshall WF (2010) Building the centriole. Curr Biol 20: R816-825.
4. Bornens M (2012) The centrosome in cells and organisms. Science 335 422-426.

5. Tsang WY, Dynlacht BD (2013) CP110 and its network of partners coordinately regulate cilia assembly. Cilia 2: 9.

6. Kobayashi T, Dynlacht BD (2011) Regulating the transition from centriole to basal body. J Cell Biol 193: 435-444.

7. Bärenz F, Mayilo D, Gruss OJ (2011) Centriolar satellites: busy orbits around the centrosome. Eur J Cell Biol 90: 983-989.

8. Kubo A, Sasaki H, Yuba-Kubo A, Tsukita S, Shiina N (1999) Centriola satellites: molecular characterization, ATP-dependent movement toward centrioles and possible involvement in ciliogenesis. J Cell Biol 147: 969-980.

9. Nigg EA, Raff JW (2009) Centrioles, centrosomes, and cilia in health and disease. Cell 139: 663-678.

10. Nigg EA (2002) Centrosome aberrations: cause or consequence of cance progression? Nat Rev Cancer 2: 815-825.

11. Bettencourt-Dias M, Hildebrandt F, Pellman D, Woods G, Godinho SA (2011) Centrosomes and cilia in human disease. Trends Genet 27: 307-315

12. Satyanarayana A, Wiemann SU, Buer J, Lauber J, Dittmar KE, et al. (2003) Telomere shortening impairs organ regeneration by inhibiting cell cycle reentry of a subpopulation of cells. EMBO J 22: 4003-4013.

13. Schmitt CA, Fridman JS, Yang M, Lee S, Baranov E, et al. (2002) A senescence program controlled by p53 and p16INK4a contributes to the outcome of cancer therapy. Cell 109: 335-346.

14. Pazolli E, Stewart SA (2008) Senescence: the good the bad and the dysfunctional. Curr Opin Genet Dev 18: 42-47.

15. Steuerwald NM, Steuerwald MD, Mailhes JB (2005) Post-ovulatory aging of mouse oocytes leads to decreased MAD2 transcripts and increased frequencies of premature centromere separation and anaphase. Mol Hum Reprod 11: 623-630.

16. Ohshima S, Seyama A (2010) Cellular aging and centrosome aberrations Ann N Y Acad Sci 1197: 108-117.

17. Erceg P, Milosevic DP, Despotovic N, Davidovic M (2007) Chromosomal changes in ageing. J Genet 86: 277-278.

18. Wojda A, Witt M (2003) Manifestations of ageing at the cytogenetic level. $J$ Appl Genet 44: 383-399.

19. Estrada JC, Torres Y, Benguría A, Dopazo A, Roche E, et al. (2013) Human mesenchymal stem cell-replicative senescence and oxidative stress are closely linked to aneuploidy. Cell Death Dis 4: e691.

20. Harley CB, Futcher AB, Greider CW (1990) Telomeres shorten during ageing of human fibroblasts. Nature 345: 458-460.

21. Martens UM, Chavez EA, Poon SS, Schmoor C, Lansdorp PM (2000) Accumulation of short telomeres in human fibroblasts prior to replicative senescence. Exp Cell Res 256: 291-299.

22. Hemann MT, Strong MA, Hao LY, Greider CW (2001) The shortest telomere, not average telomere length, is critical for cell viability and chromosome stability. Cell 107: 67-77.

23. Parrinello S, Samper E, Krtolica A, Goldstein J, Melov S, et al. (2003) Oxygen sensitivity severely limits the replicative lifespan of murine fibroblasts. Nat Cell Biol 5: 741-747.

24. Busuttil RA, Rubio M, Dollé ME, Campisi J, Vijg J (2003) Oxygen accelerates the accumulation of mutations during the senescence and immortalization of murine cells in culture. Aging Cell 2: 287-294.

25. Robles SJ, Adami GR (1998) Agents that cause DNA double strand breaks lead to p16INK4a enrichment and the premature senescence of normal fibroblasts. Oncogene 16: 1113-1123.

26. Di Leonardo A, Linke SP, Clarkin K, Wahl GM (1994) DNA damage triggers a prolonged p53-dependent G1 arrest and long-term induction of Cip1 in normal human fibroblasts. Genes Dev 8: 2540-2551.

27. Atadja P, Wong H, Garkavtsev I, Veillette C, Riabowol K (1995) Increased activity of p53 in senescing fibroblasts. Proc Natl Acad Sci U SA 92: 8348-8352.

28. Lin AW, Barradas M, Stone JC, van Aelst L, Serrano M, et al. (1998) Premature senescence involving p53 and p16 is activated in response to constitutive MEK/MAPK mitogenic signaling. Genes Dev 12: 3008-3019. 
Citation: Hossain D, Tsang WY (2013) Centrosome Dysfunction and Senescence: Coincidence or Causality? Aging Sci 1: 113. doi: 10.4172/23298847.1000113

29. Serrano M, Lin AW, McCurrach ME, Beach D, Lowe SW (1997) Oncogenic ras provokes premature cell senescence associated with accumulation of p53 and p16INK4a. Cell 88: 593-602.

30. Zhu J, Woods D, McMahon M, Bishop JM (1998) Senescence of human fibroblasts induced by oncogenic Raf. Genes Dev 12: 2997-3007.

31. Miao YL, Sun QY, Zhang X, Zhao JG, Zhao MT, et al. (2009) Centrosome abnormalities during porcine oocyte aging. Environ Mol Mutagen 50: 666-671.

32. Eichenlaub-Ritter U, Chandley AC, Gosden RG (1986) Alterations to the microtubular cytoskeleton and increased disorder of chromosome alignment in spontaneously ovulated mouse oocytes aged in vivo: an immunofluorescence study. Chromosoma 94: 337-345.

33. Eichenlaub-Ritter U, Stahl A, Luciani JM (1988) The microtubular cytoskeleton and chromosomes of unfertilized human oocytes aged in vitro. Hum Genet 80: 259-264.

34. Miao YL, Kikuchi K, Sun QY, Schatten H (2009) Oocyte aging: cellular and molecular changes, developmental potential and reversal possibility. Hum Reprod Update 15: 573-585

35. Schatten H, Chakrabarti A, Hedrick J (1999) Centrosome and microtubule instability in aging Drosophila cells. J Cell Biochem 74: 229-241.

36. Manning JA, Kumar S (2010) A potential role for NEDD1 and the centrosome in senescence of mouse embryonic fibroblasts. Cell Death Dis 1: e35.

37. Mikule K, Delaval B, Kaldis P, Jurcyzk A, Hergert P, et al. (2007) Loss of centrosome integrity induces p38-p53-p21-dependent G1-S arrest. Nat Cell Biol 9: 160-170

38. Srsen V, Gnadt N, Dammermann A, Merdes A (2006) Inhibition of centrosome protein assembly leads to p53-dependent exit from the cell cycle. J Cell Bio 174: 625-630.

39. Schmidt S, Schneider L, Essmann F, Cirstea IC, Kuck F, et al. (2010) The centrosomal protein TACC3 controls paclitaxel sensitivity by modulating a premature senescence program. Oncogene 29: 6184-6192.
40. Huck JJ, Zhang M, McDonald A, Bowman D, Hoar KM, et al. (2010) MLN8054, an inhibitor of Aurora A kinase, induces senescence in human tumor cells both in vitro and in vivo. Mol Cancer Res 8: 373-384.

41. Katayama H, Sasai K, Kawai H, Yuan ZM, Bondaruk J, et al. (2004) Phosphorylation by aurora kinase $\mathrm{A}$ induces Mdm2-mediated destabilization and inhibition of p53. Nat Genet 36: 55-62.

42. Ohshima S (2012) Centrosome aberrations associated with cellular senescence and p53 localization at supernumerary centrosomes. Oxid Med Cell Longev 2012: 217594.

43. Oricchio E, Saladino C, lacovelli S, Soddu S, Cundari E (2006) ATM is activated by default in mitosis, localizes at centrosomes and monitors mitotic spindle integrity. Cell Cycle 5: 88-92.

44. Tritarelli A, Oricchio E, Ciciarello M, Mangiacasale R, Palena A, et al. (2004) p53 localization at centrosomes during mitosis and postmitotic checkpoint are ATM-dependent and require serine 15 phosphorylation. Mol Biol Cell 15: 3751-3757.

45. Ciciarello M, Mangiacasale R, Casenghi M, Zaira Limongi M, D'Angelo M, et al. (2001) p53 displacement from centrosomes and p53-mediated G1 arrest following transient inhibition of the mitotic spindle. J Biol Chem 276: 19205 19213.

46. Morris VB, Brammall J, Noble J, Reddel R (2000) p53 localizes to the centrosomes and spindles of mitotic cells in the embryonic chick epiblast, human cell lines, and a human primary culture: An immunofluorescence study. Exp Cell Res 256: 122-130

47. Tarapore P, Tokuyama Y, Horn HF, Fukasawa K (2001) Difference in the centrosome duplication regulatory activity among p53 'hot spot' mutants: potential role of Ser 315 phosphorylation-dependent centrosome binding of p53. Oncogene 20: 6851-6863.

48. Shinmura K, Bennett RA, Tarapore P, Fukasawa K (2007) Direct evidence for the role of centrosomally localized p53 in the regulation of centrosome duplication. Oncogene 26: 2939-2944. 